\title{
Integrating Behavioural Animation Techniques
}

\author{
Jean-Sébastien Monzani, Angela Caicedo and Daniel Thalmann ${ }^{\dagger}$ \\ Computer Graphics Laboratory, Swiss Federal Institute of Technology, Lausanne, Switzerland \\ http://ligwww.epfl.ch/
}

\begin{abstract}
Our research focuses on animating autonomous virtual humans which are able to take decisions by themselves. We especially address in this paper the technical problem of integrating altogether the physical simulation of agents (represented as virtual humans in a 3D environment) and their behaviours and motivations, driven by a Beliefs, Desires and Intentions architecture. We also explain how goals drive plans, and how an agent can coherently handle concurrent tasks.
\end{abstract}

Keywords: behavioural animation and planning, virtual humans animation, inter-agents communication

\section{Introduction}

Virtual humans are commonly used nowadays in the entertainment industry, and most specifically in movies and video games. If the quality of pictures has been dramatically improved during the last years, the animation is still a major bottleneck in production. For movies, one can afford to spend months in order to produce a realistic animation, but for real-time applications (and this is particularly true in video games) it is still very difficult to handle the behaviour of virtual agents, especially when we try to make them more autonomous. Consequently, dedicated tools like the Improv system ${ }^{15}$ from Ken Perlin or commercial applications, such as Motion Factory's Motivate ${ }^{14}$ or Virtools' $\mathrm{NeMo}^{18}$ have tried to help the animator in the creation of basic behaviours. Behavioural Animation has numerous advantages: virtual humans acquire the capabilities of perceiving their environment and are able to react and take decisions, depending on this input. Autonomous agents are more flexible, and consequently produce less repeating animation patterns, as we still often see in video games based on actions/reactions schemes.

The major challenge now is to integrate into the same framework a set of various techniques which are required for simulating behaviours of virtual humans. This includes the $3 \mathrm{D}$ rendering and animation, motion control, action selection (using goals and plans for instance) and simulated

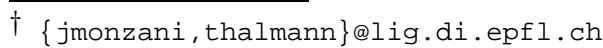

(c) The Eurographics Association and Blackwell Publishers 2001. Published by Blackwell Publishers, 108 Cowley Road, Oxford OX4 1JF, UK and 350 Main Street, Malden, MA 02148, USA. inter-agents verbal communication. It is indeed a very exciting issue, but the integration is not simply a matter of taking the pieces of a puzzle and putting them altogether. It is crucial to develop an architecture which is able to support and handle a lot of concurrent tasks. The smart avatars of Bindiganavale $e t a .^{2}$ are pretty similar to what we are trying to develop. They are using the Parameterised Action Representation (PAR) for handling action selection and animation. PARs can represent a primitive or a complex action, and are able to control the motion generators of the virtual humans. By adding a Natural Language translator, one can even give orders in English which are then converted into PARs. We were not concerned by the issue of natural language translation, and our system is not competitive regarding this field. Unfortunately, there are not that much integrated applications which are trying to reach the same goal as us. This is why we are now going to present some major contributions in various domains including both virtual humans animation and behavioural techniques.

Starting with the animation of virtual humans, one of the first difficulties is to be able to control concurrent motions. Granieri's Parallel Transition Networks ${ }^{9}$ is one solution which is considering the various processes involved in a motion as a network into which one can include predicates and conditions. Motivate's ${ }^{14}$ approach relies on dynamic event-based behaviour programming where simple behaviours can be combined into networks and graphically describe behaviours and motions. It also uses a specific 
scripting language, just as Improv ${ }^{15}$ does. We also adopt a scripting language and extend it to match our needs.

Regarding the specific case of gestures involved in virtual humans conversation, Cassel et al ${ }^{8}$ studied an automatic generation of movements and facial expressions (during conversation), based on the content of the dialog itself. An other contribution is the interactive story-telling with $2 \mathrm{D}$ cartoonlike characters, as demonstrated in the Oz Project ${ }^{12}$.

Finally, an autonomous agent has to select its actions by itself. Research has been driven by people from different areas: ethologists such as Tinbergen ${ }^{19}$, and computer scientists such as Brooks ${ }^{6}$, Maes ${ }^{11}$ and Minsky ${ }^{13}$ who lead the school of Behaviour-Based Artificial Intelligence (BBAI). Our model, as proposed in the BBAI, does not attempt to build models of the world, and the agent has to reevaluate its course of action on every slot of time. Some points are not directly addressed by the BBAI such as the interplay between internal factors (such as emotional levels) and external factors (common world situations).

We are now going to present the motivations that pushed the design of our system. Especially, the separation of simulated physical functionalities (low-level modules) and the logical management of behaviours (high-level modules). The next section describes the scenario that we chose to demonstrate the capabilities of the system and we will show how decisions are taken by agents through plans. We will continue with the presentation of animation concepts for mixing concurrent gestures and finally describe results before concluding.

\section{System presentation}

\subsection{Why separate the body and the brain?}

Automatically animating a virtual human is such a complex task, that one has to decompose it into simpler elements. In this paper, we will use the terms of low and high-levels. Saying that a task belongs to low level does not means that it is something easier or faster to achieve! We will rather regroup in the low-level the physical elements (for the virtual human, this includes the body, and basic animations like locomotion or objects interaction), and the behaviour will be handled by the high-level, simulating the virtual human's brain.

The low-level structures are responsible for physical elements that include: the 3D graphical representation of virtual humans or objects, their animation and the simulation of sound. Virtual humans are able to interact with objects, or with other humans (for instance, giving a paper to someone else). They also communicate verbally, and naturally exchange greetings and information with artificial speech. On the other side, high-level modules give to the agent some $b e$ liefs (about itself, others or the environment) and depending on personal needs, the agent tries to fulfil its goals through the help of provided plans. Of course, both low and highlevels are interconnected so that the agent's behaviour can be reflected physically (walking, for instance) and similarly, if something changes in the environment (like someone speaking), the low-level will warn the high-level of the event, just as our brain and body are working altogether.

Such a low/high level separation is helpful. Imagine that you have to come out and go to the train station. Would you really take care of each of your subtle motions, watch your steps, or even notice that you are avoiding other people? In your mind, you just have the goal of going to the station: this is your high level decision. The low level walking motion with obstacle avoidance is a very complex task, but you do not notice it. This simple example clearly demonstrates how useful it is to separate the behaviour from the motion.

As we will see later on, this separation greatly helps to focus on behaviours without having to deal with low-level considerations. Furthermore, it is then easier to test, maintain and extend code: either when implementing a new kind of facial expression or when creating new behavioural plans, one concentrate on the animation factors or simply ignore the physical aspects and test the plans without a graphical feedback.

Regarding the programming languages, the graphical representation of the environment is a CPU intensive task and is therefore using $\mathrm{C}++$ for maximal performance. For animation, it is easier to avoid compilation and enter interactive commands: we thus adopted the scripting language Python ${ }^{16}$ as it is easy to integrate it with the $\mathrm{C}++$ layer, and offers a powerful set of functionalities. Maybe one of the most important useful features are its capabilities for creating threads, which allow us to handle concurrent inputs from the environment. It is also very convenient for merging gestures at the same time, e.g. by using one thread for animating the head, and one for legs motion. Regarding the decisionmaking process, we primarily used LISP and then switched to Java for efficiency and better code support. As you will see in the next section, it was easy to exchange these languages since the communication between the low and high levels is performed through TCP/IP.

\subsection{System design}

The previous considerations in low and high level separation have led to the construction of our system, as depicted in figure 1. We are now going to present the low-level components (Agents' Common Environment, from hereby referenced as ACE) and the high-level decisions making (the Intelligent Virtual Agent or IVA).

\subsubsection{Agents' Common Environment (ACE)}

To simulate the physical word, we have developed a system called ACE for Agents' Common Environment. It comprises a set of different commands for controlling the simulation, like the creation and animation of 3D objects, virtual humans, and smart objects ${ }^{10}$. Virtual humans encapsulate various motion motors and may have facial expressions. They 


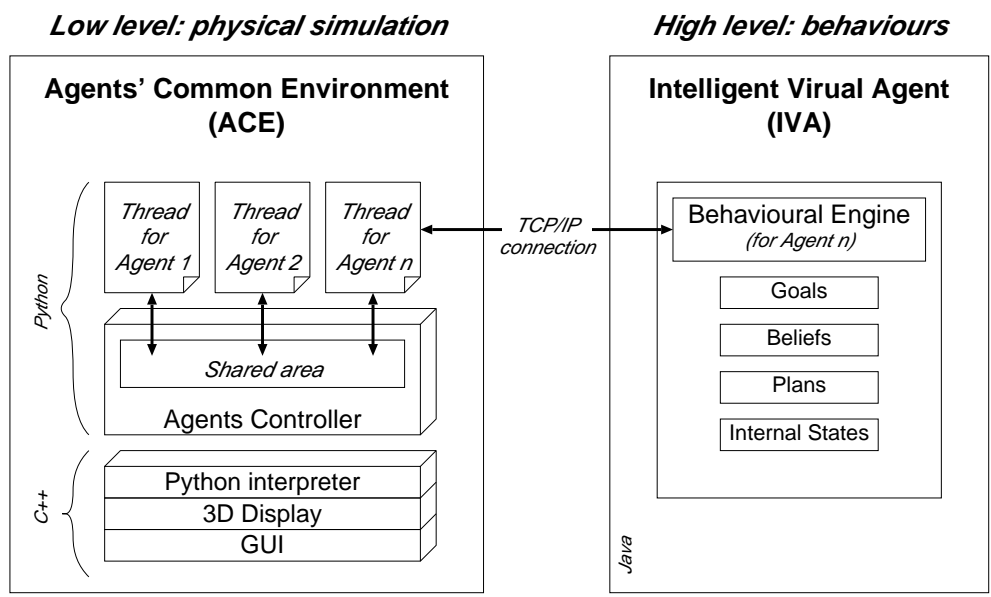

Figure 1: Low and high level components in the system

are able to replay a recorded animation (key-frames animation), can walk, or use Inverse Kinematics ${ }^{1}$ for better interactions with objects. Finally, they can visually perceive their environment ${ }^{4}$.

ACE is mainly coded in $\mathrm{C}++$ to ensure high performances. For convenient user-interaction, it also provides a Python layer which interprets commands on the fly and animates the virtual humans. Python is an all-purpose scripting language that we have extended to fit our needs. More precisely, when the application is launched, a simple environment is created and displayed in a window, and a command shell is prompted, ready for entering commands in Python. This way. ACE provides the basic commands for loading, moving, animating humans and objects, giving a powerful set of functionalities straight from the scripting language. It is very convenient indeed to reuse a language and extend it to match our purposes, rather than developing a new syntax from scratch: this saves time and gives the opportunity to reuse third-party modules, which have been already implemented and tested by others.

In ACE, each agent is running in a separated process or thread, so that they can independently interact with the 3D environment. When we want to create an agent, we code the corresponding Python script and load it into the Agents Controller: this layer, added on top of ACE, provides a shared area which helps the inter-agents communication and synchronisation with the environment. Otherwise, each agent would be let on its own, with no way to know what others are doing, or when they are speaking, for instance. This shared area is especially useful for verbal inter-agents communication. The design of agents will be discussed in section 2.3.

\subsubsection{Intelligent Virtual Agent (IVA)}

The Intelligent Virtual Agent relies on a Beliefs, Desires and Intentions architecture, as described by Georgeff ${ }^{17}$, so that each agent manages its own beliefs and makes decisions by itself. While agents in the low-level have to share a common area and are handled by the Agents Controller, there is no such need for the IVA. Therefore, even if each IVA runs into a separated Java thread over the same application, there is no inter-threads communication (figure 1 only shows one IVA for Agent $n$ ). This is in accordance with real human interaction: we do not have direct connections between our brains, but we need to use our body for communicating.

The knowledge of the agent is decomposed into its beliefs and internal states (like the anxiety for instance), the goals to achieve, and the plans, which specify a sequence of actions required to achieve a specific goal. Based on these, the agent is then able to select the correct actions to perform in order to achieve its goals. Beliefs and goals may evolve over time, and the agent is able to take into account new elements, like elements changing in the environment and dynamically react. We will describe in section 3.3 how plans are working; for more details on the Intelligent Virtual Agent, one can refer to a previous article . $^{7}$.

\subsubsection{Interconnecting Low and High-Levels}

By using sockets and TCP/IP connection, the system can run in a distributed way, reducing the $\mathrm{CPU}$ cost on the machine which is responsible for the $3 \mathrm{D}$ environment display. A connection is established between each ACE agent thread and the corresponding IVA. It is then pretty easy for the IVA to send orders to the low-level (like, making the agent move, or speak). Similarly, incoming input (like a verbal message) will be carried and sent by the low-level to the high-level behavioural module. This is demonstrated in figure 2: when Agent 1 speaks to Agent 2, the message goes first to the lowlevel, then the thread for the first agent puts the message in the shared area. Agent 2 is then able to retrieve the message and hear it. 
High level: IVAs

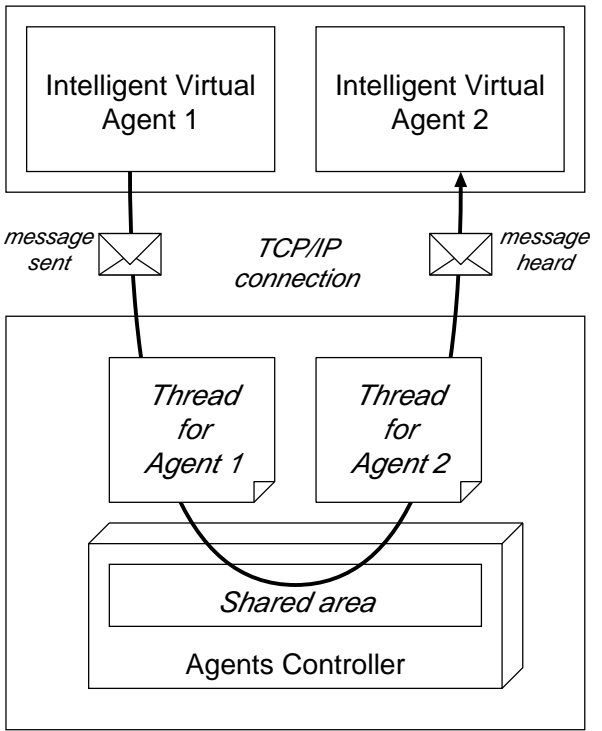

Low level: $A C E$

Figure 2: Verbal communication between two IVAs has to go through the low-level

\subsection{Agent design and capabilities}

The physical representation of the agent should handle various capabilities, such as: perception, verbal communication, performing actions and connecting to the IVA behavioural module. In OOP, an elegant approach is to split each capability into one class and merge all of them into the definition of what an agent should be able to do with class inheritance. Using $\mathrm{UML}^{3}$, we present in figure 3 the definition of the Agent class, as implemented in Python.

ACE functions refer to agents as unique ID, so, we start by defining the AgID class as a super class, sharing the ID among the inherited classes. From this, we derive the AgThread, so that agents can run their own code in a separated process (with the help of the standard Thread class). As we have seen before and depicted on the figure, threads run under the responsibility of the Agents Controller. Regarding the capabilities, the AgPerceive class encapsulates all the methods that allow the agent to visually perceive objects and remembers when objects get into or out of focus. AgTalking is responsible for the inter-agents communication and helps the agent to talk and hear other agents. The final Agent class inherits from these three basic classes, which means that our Agent is able to speak to someone, hear when someone speaks and perceive the objects in the environment.

The Agent also references the TasksHandler object. This class is in charge of handling parallel tasks like walking, looking, playing keyframes, applying facial expressions or interacting with objects. This will be discussed in section 4 . Finally, the AgSocket connects the agent to its IVA and is able to decode orders coming for the IVA or send stimuli like visual perception back to it.

\section{Making them move}

\subsection{Choosing a scenario}

Features of a behavioural animation system can only be demonstrated in a carefully chosen scenario. As depicted in figure 4, we simulate an office in which employees are editing a journal. Since people are working, they do endow some roles and this influences their ability to perform or delegate jobs. At the top of the hierarchy is the Editor. He chooses stories or events to investigate, and asks the Journalist to write down a story and take photos. When the Journalist comes back, he gives the corresponding story and films to the Secretary, who then delegate the correction to the Text corrector and the design of illustrations to a Graphic designer. When everything is ready, the Page designer creates a draft version of the article and sends it back to the Editor for approval.

This scenario has a lot of potential inter-agents cooperations and interactions: they have to speak, give and pick up some objects, and interact with elements such as computers on the desks. Depending on their roles, they can delegate jobs. As they can only perform one job at a time, they have to suspend an on-going job if a more important job comes along. Finally, it is possible to modify some elements during the simulation (like the time taken to complete a job) and see how agents react.

Figure $5^{*}$ shows a snapshot of the running simulation with three agents in focus. The window on the left gives access to the Python commands and to other tools such as positioning objects, controlling the camera, and so on.

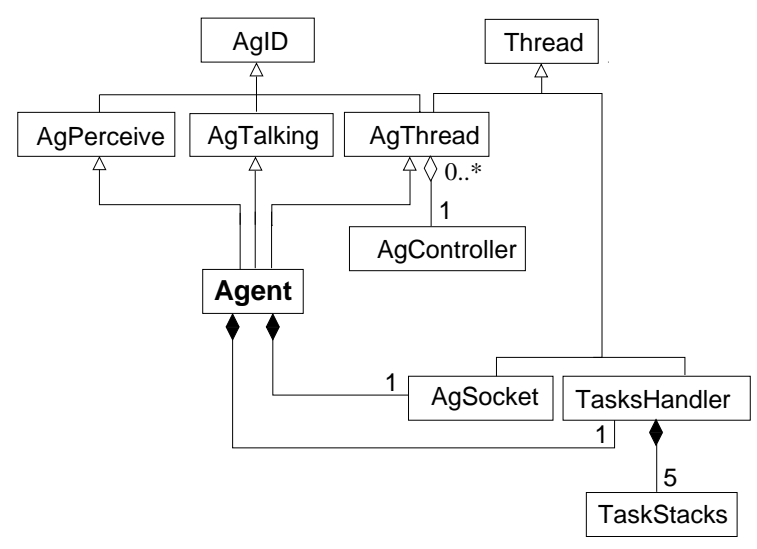

Figure 3: Multiple inheritance architecture defining the agent capabilities 
3.2. Visual and auditive feedback: gestures, interactions and sound

The powerful notion of smart objects ${ }^{10}$ is used to simulate human-objects interactions. Smarts objects do not only contain the graphical representation of objects in the virtual environment, but also include a description of their functionalities, and how these functionalities can be used. These interaction behaviours are then available for each virtual human: it just has to come to the object which is then able to "guide" it on how to perform the action. For instance, if a virtual human wants to take the elevator, the smart object decomposes the actions of pressing the button to call the elevator, then entering into the lift, selecting the floor and finally going out.

When we designed our scenario, we came across the problem of exchanging objects. We wanted to use a simple func-

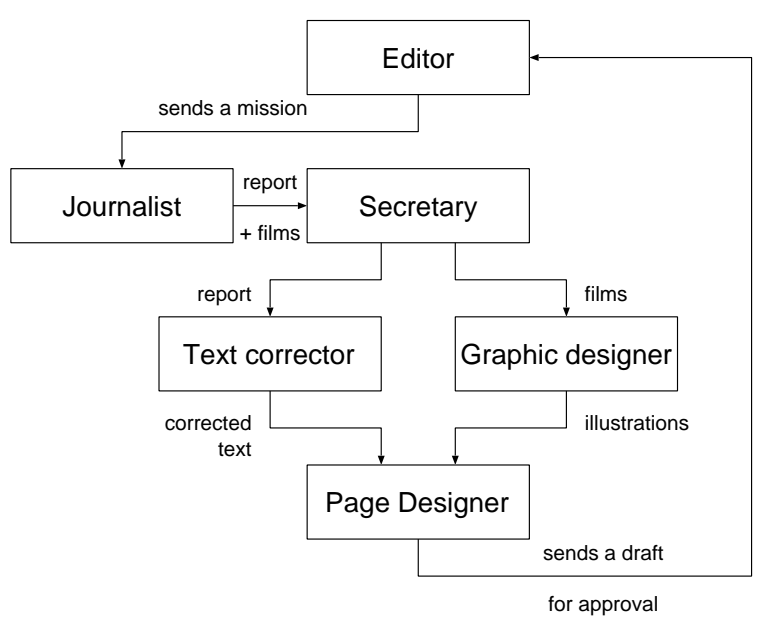

Figure 4: Roles in the edition of the newspaper

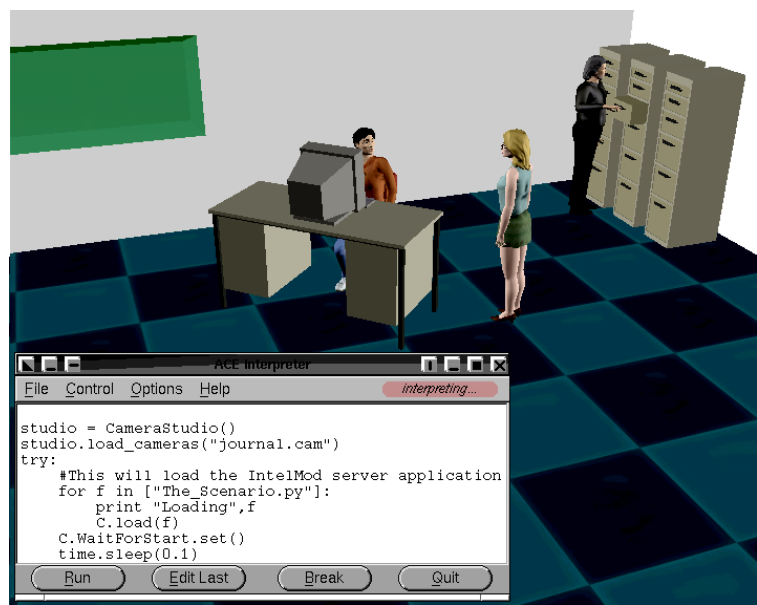

Figure 5: The environment with three agents. The window on the left is for editing Python scripts. tion so that on agent is able to give something to another one. We solved this issue by creating a special smart object called Exchanger. It is indeed an invisible object which supports put and get methods. When an agent wants to perform an exchange at a certain location, we create an Exchanger here and drive the receiver agent there. It is then easy for the first agent to put the object to be retrieved by the other one. By synchronising the agents accordingly, we also avoid having an object which is suddenly "floating" in the space during the transaction. The notion of Exchanger can also be extended so that exchanged objects like papers are stacked up on a desk. When the agent has to do the job, it picks up the correct paper and reads it.

The actual production of sound through speech synthesis also makes the demonstration more attractive. Since our goal is not to produce realistic speech but just to enhance the simulation by adding sound, we did not try to develop a module on our own, but integrated instead an available package called MBROLA (designed by the Speech Synthesis Group at TCTS Lab, Belgium). By adding an emotion filter, we are also able to alter the sound in order to reflect simple emotions such as fear, for example.

\subsection{Defining behaviours with plans}

\subsubsection{Overview}

An agent determines its behaviour by reasoning about what it knows to be true at a specific time. Into the classical planning systems, plans provide step by step instructions on how to satisfy some goals; they basically require some preconditions to be fulfilled in order to activate their effects. For instance, if the agent is thirsty, it has to drink. But before drinking, it should pick-up a glass and fill it with water. For an introduction to these concepts, one can refer to the work of Maes ${ }^{11}$.

To make plans more generic, we also adopted the common approach of pattern matching: a parser is responsible for instantiating variables so that the same plan can be reused for different cases. One should notice that symbols beginning with a question mark will be instantiated by the pattern matcher. For instance working-on CorrectingText from Secretary will match working-on ?prevjob from ?prevboss. Furthermore, the user can add his/her own internal parameters in order to model continuous values which can be altered dynamically. For instance, the happiness of the agent might range from -1 (unhappy) to 1 (happy). This integration of internal states is very effective for difficult situations (which could not be solved with pattern matching only).

Apart from its name and the required preconditions, a plan is composed of internal states than can be tested after the preconditions. If both preconditions and internal states are fulfilled, the effects are executed. They are basically of four types: adding or deleting beliefs from the agent's beliefs, 
changing the internal states, and sending orders to the lowlevel (animations, gestures, speech, etc).

To help their management, plans can be categorised and regrouped. Apart from agent specific plans (that is, plans that are only available for one agent, depending on its abilities), we introduce plans for job execution and delegation, plans for displacements into the environment and for interagents communication. These plans make the virtual humans behave in a socially correct way: for instance, agents will exchange greetings before starting a conversation (see section 3.3.3). Or, they can suspend the job that they are doing currently to favour a more important one. Of course, if one wants to simulate an agent which is not as polite as the others, it is easy to remove some of these plans and simulate a rude person.

Before continuing with a presentation of a sample plan, we have to add that it is crucial to be able to monitor the evolution of beliefs and goals for each agent: without the help of a dedicated graphical user interface, agents are behaving by themselves with no control over what their motivations are. This approach is good for demonstrations but unacceptable when developing.

\subsubsection{Example}

Figure 6 shows a complex plan: giving the fact that our agent is working on a job assigned by someone, if someone else comes with a new and more important job, then the previous job will be suspended to favour the new one.

From the agent's point of view, this can be summarised by "If I'm working on the job named ?prevjob (assigned by ?prevboss) and if ?boss asks me to do a new ? job, I will suspend my previous job ?prevjob and take the new one if this new job is more important than my current one."

In order to model this, the agent needs to know the importance of everyone in the company. This is stored in a hash table hierarchy from which we retrieve a number representing the person's importance. Consequently, when the agent is working, this number is stored in the state current_job_priority to reflect the importance of the job. It is then easy to test this value when someone comes with a new job, as reflected in the states part of the plan.

To briefly describe the plan, the preconditions can first be translated to "I'm working on a job and someone else is coming with a new job.". As we have seen, the internal states are then evaluated to see if the incoming plan is more important than the current one. If so, the agent deletes and adds some beliefs so that it suspends the previous job and takes the new one. Consequently, two new goals are set: the need to suspend the previous job (?prevjob), and the need to perform the new one (?job). The current_job_priority is of course updated accordingly (Eval statement). Finally, one can notice that a verbal communication will be established between the agents, so that the ?boss is informed of the decision taken by the agent. An order to say something in sent to the low-level (latest statement Add (say ...)).

It is interesting to notice that the verbal communication does not rely on natural language analysis: it would be a waste of time to compute a translation from the representation of beliefs to natural language, transmit the text in English and then compute the reverse transformation. To avoid this, we split the message into the semantic and the verbal realisation of the sentence: the pattern for speech act is of the form (say agent semantic sentence). The semantic will be transmitted to agent and usually added to its beliefs, while the sentence is pronounced by the speech generation system. The semantic (informed ?self working on ?job) is using the special variable ?self which is always replaced by the name of the agent who is executing the plan. If the Graphic designer warns the Secretary that he is working on some illustrations, then the Secretary receives (informed Graphics-Designer working on Illustrations). There is one drawback to this approach, though: due to the separation of the semantic and the verbal realisation of the message, the designer of the plans has to carefully maintain the coherence between them.

\subsubsection{Dialog plans}

Figure 7 presents how a message is sent when conversing: added beliefs are represented here with the notation + belief and removed ones with - belief, all depending on the triggered plan. Plans for conversation are designed so that if an agent needs to ask something, it has to perform some greetings first. To demonstrate how plans are triggered, imagine that an agent name Query $(Q)$ is about to send a QUERY to the agent Answer (A). Before starting the conversation, Query adds two beliefs: (i) that it needs to say QUERY to Answer and (ii) it needs also to talk to Answer. If agents are not conversing yet, the second belief is triggering the conversation process, as represented in step 1: assuming that Query needs to talk to Answer and that it is not conversing yet, Query sends the message HELLO to Answer to initiate the conversation process. Consequently, during step 2, Answer receives this HELLO message since it is not conversing yet with Query, it sends back a HELLO message, in order to warn $\mathrm{Q}$ that it is now ready to converse. Now, both agents have exchanged greetings and are aware that they are conversing (step 3). During step 4, Query can now send its QUERY to Answer and Answer can answer back (this is not shown here). When Query's Behavioural Engine decides that it has nothing more to ask, it removes the need to talk to Answer, and this triggers the sending of the BYE message to Answer step 5. Finally, Answer also stops the conversation by sending BYE to Query (step 6). 


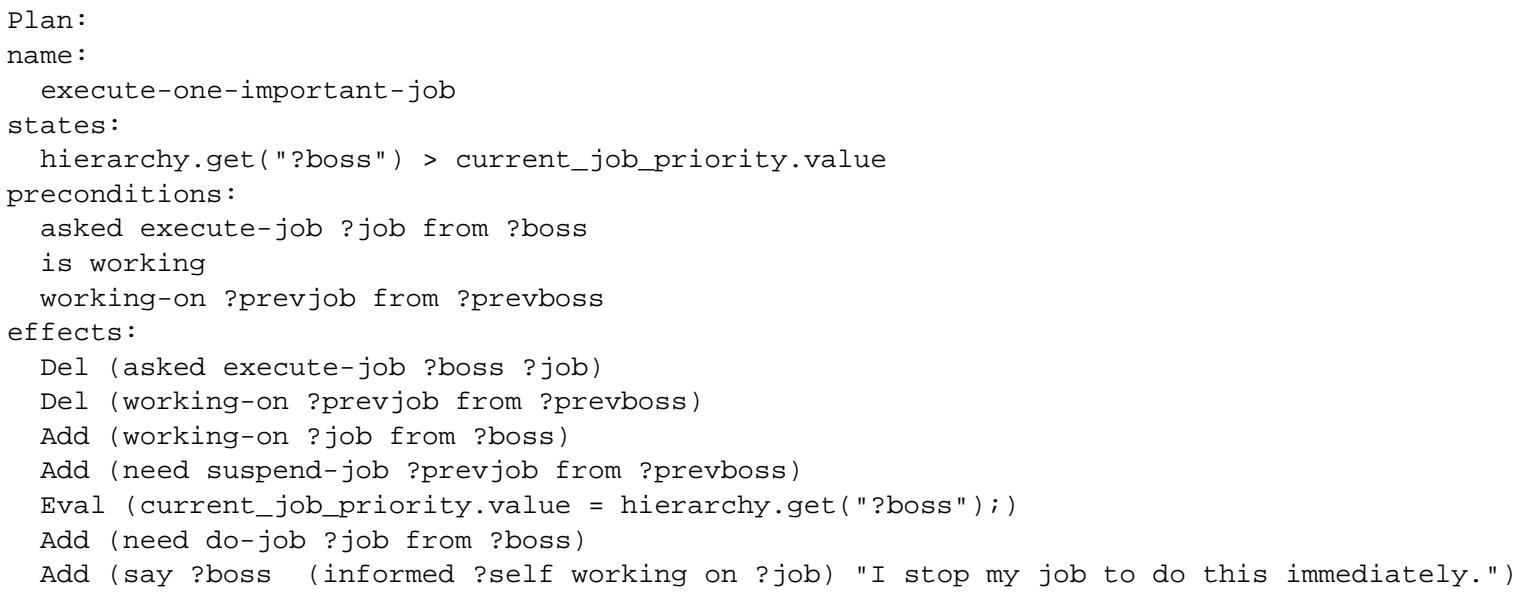

Figure 6: Example of plan

\section{Low-level 3D animation using Tasks}

We propose a layered architecture to help animation of virtual humans: Actions provide basic behaviours such as walk, look and coherently mix them. Tasks and Tasks Stacks ease the automatic activation and inhibition of actions, all under the responsibility of the Tasks Handler.

\subsection{Actions}

Actions are the simplest way to control the posture of the virtual human. They can be either activated or not, and smooth transitions between these states are computed by adjusting the individual actions weights. The library included in $\mathrm{ACE}^{5}$ can animate and coherently combine walking, looking, object interaction, facial expression and replay of recorded keyframe sequences.

This approach is elegant and produces smooth animations, but is unfortunately not sufficient to specify high level behaviours, as every action has to be triggered individually. For instance, sequencing actions like "do action 1 then do action 2" requires to check when action 1 is finished, then remove it and activate action 2. Therefore, for something as simple as following a path (which we decompose into go to location 1, then go to location 2, etc...), one has to check individually each location. We experimented that this becomes quickly complicated and we introduced Tasks to deal with this problem.

\subsection{Combining Actions into Tasks}

Tasks are a convenient way to execute actions and monitor their evolution over time. They are implemented as Python classes, and all inherit from the same generic task which contains the task callback: this is a reference to one or more actions that should be executed when the Task is activated. Similarly, the termination callback checks regularly if the

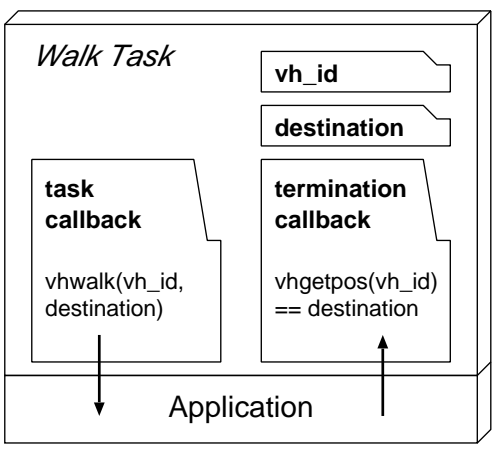

Figure 8: Example callback for a walking task

Task is terminated and if so, the Task will be automatic removed from its Tasks stack, as we will see later on. Other attributes include the state of the Task (Suspended or Activated), some timing attributes and some reference to the virtual human that it is controlling (vh_id). Two important attributes are remaining: the activation which takes one of the values \{Reactivated, Repeated, Once\}, and the list of next tasks to trigger once the task is terminated. They will be presented in the next section.

As an example, figure number 8 shows some of the attributes of a Walk Task. This task first stores a reference to the virtual human which is under control (vh_id) and the destination point, which is the location that the virtual human should reach. The task callback uses the vhwalk function provided by ACE control virtual human and the termination callback regularly checks the position of the agent to see if it has arrived at the destination. 
Step 1

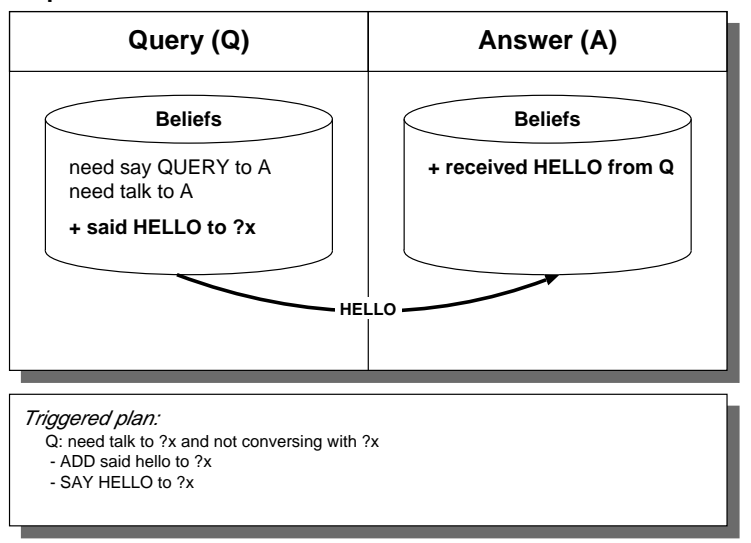

Step 3

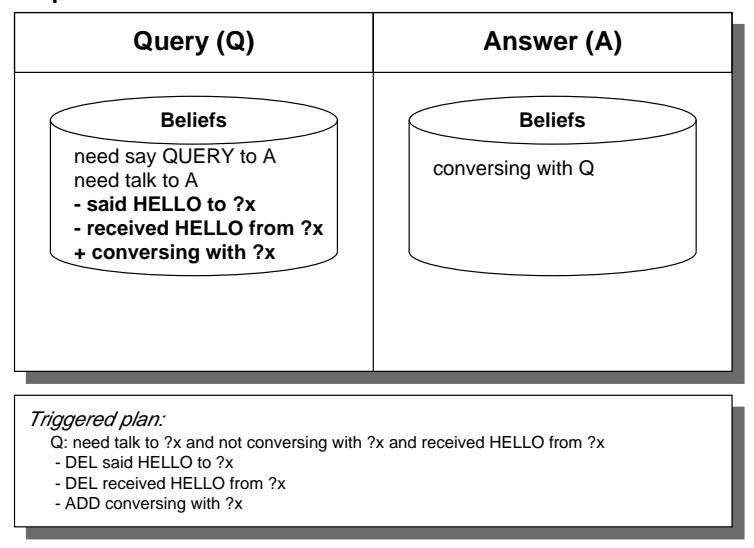

Step 5

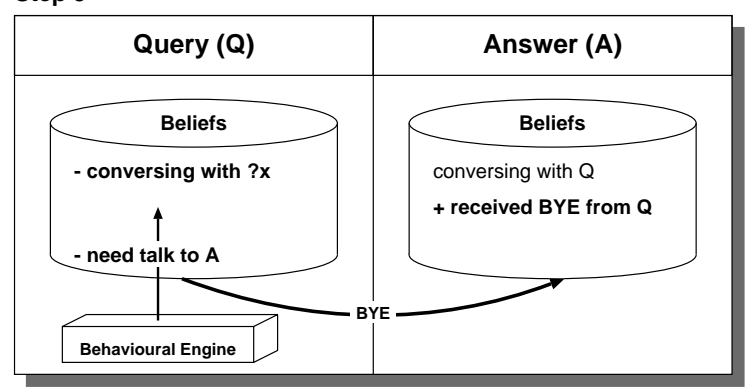

Triggered plan:

Q: not need talk to ?x and conversing with ?x

- SAY BYE to ?x

- DEL conversing with ?x
Step 2

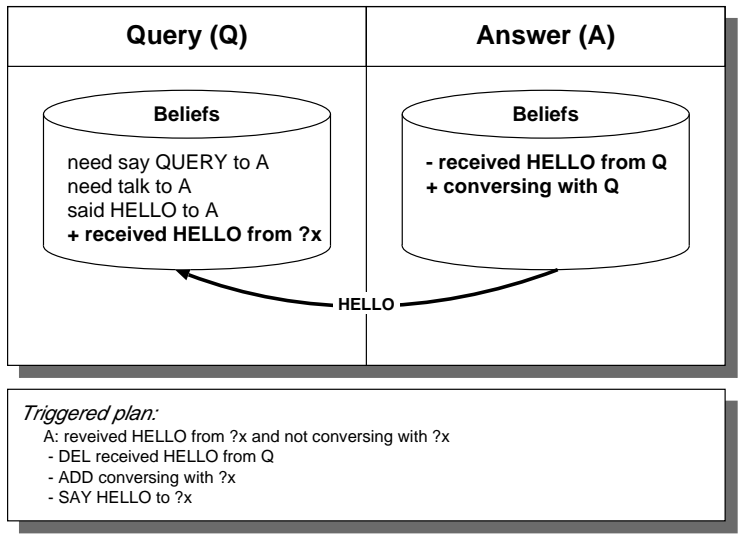

Step 4

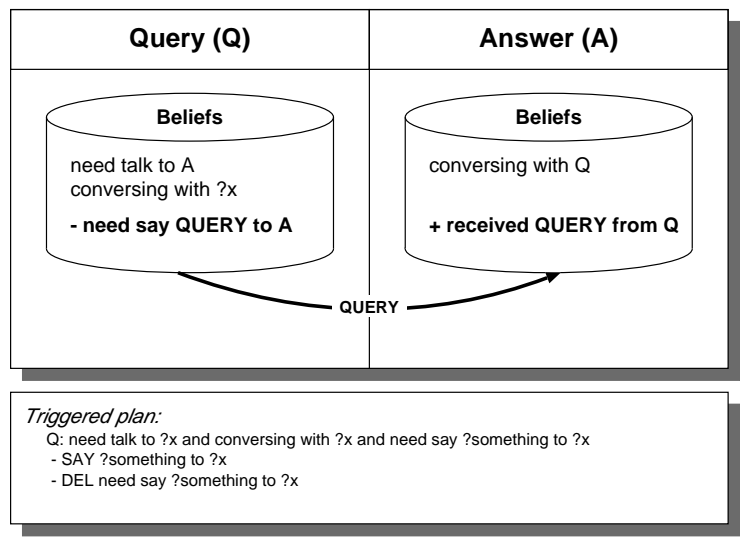

Step 6

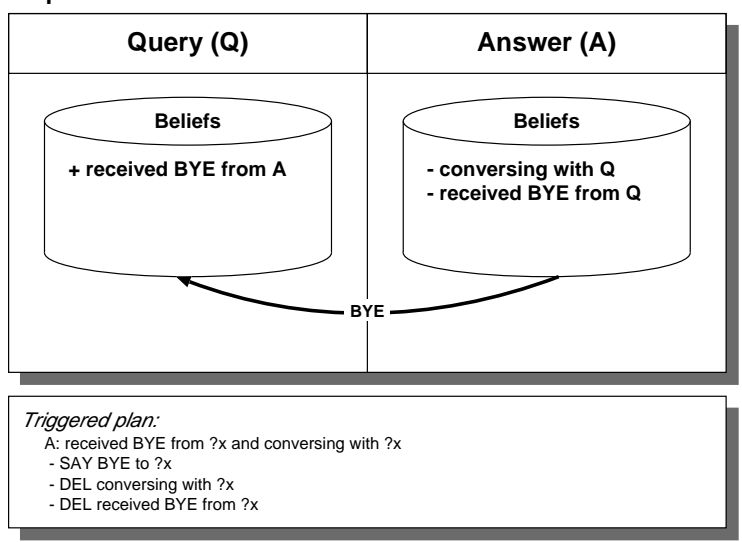

Figure 7: Example of a dialog

\subsection{Managing priorities with Tasks Stacks}

To avoid conflicts when tasks of the same type are about to control simultaneously the same elements of the body, we regroup them into stacks, with one stack of each type per agent. We are using stacks for walking, looking, interacting with objects, playing a keyframe, and manipulating the agent face. Into each stack, only one task can be executed at a specific time, and tasks on top of the stack have higher priorities than those bellow. At each frame, Tasks Stacks are 
responsible for updating Tasks, activate them, delete terminated ones, etc... Since tasks have two states (Suspended or Activated), only Activated Tasks are taken into account, as one can expect. The activation attribute of the task controls when the callback is called: if set to Once, task is activated once (playing a keyframe, for example); Repeated is for continuous tasks which should be performed at each frame (visual tracking of a moving object) and Reactivated only execute the task callback when the task becomes active.

The Task inspection algorithm for each individual Tasks Stack starts from the top of the stacks and looks for the first Activated task (Suspended ones are ignored). This task is called the top task. Then, depending on the activation of the task: if set to Once and the task has never been executed, execute it; a Repeated will always be executed. And, if the activation is set to Reactivated and the top task is not the same than for the previous frame, execute the task. Once the top task has been found, we do not execute the pending tasks anymore, but we still go through the stack in order to detect tasks which are terminated, by testing their termination callback. Terminated tasks are removed from the stack, and possibly activate other suspended tasks stored in their next tasks list.

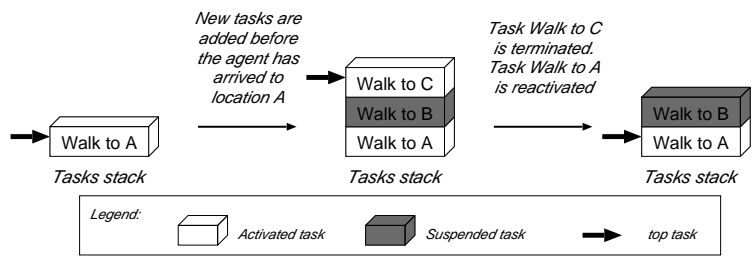

Figure 9: Reactivated tasks - Task stack for the Walking tasks

As an example of Reactivated tasks, consider figure 9: we have represented the stack of Walking Tasks for one agent. At the beginning, there is only one activated task, which asks the agent to go to location A. But before the agent could actually arrive there, two new tasks are appended on the top of the stack: one order to go to location B (which is ignored, since it is Suspended) and an order to go to location C, which becomes the top task and consequently initiates the lower level action "go to location C". When location $\mathrm{C}$ has been reached, the task is removed, and the Tasks stack reactivates "go to location A" again. Note that by default, if the agent reaches location $\mathrm{A}$ while going to location $\mathrm{C}$, then task Walk to $\mathrm{A}$ is considered to be terminated and removed from the stack. To prevent this kind of behaviour, one can suspend tasks and use the next tasks lists to activate each intermediate location when following a trajectory.

\subsection{Multiple tasks altogether: the Tasks Handler}

The Tasks Handler gathers all the Tasks stacks for one agent and repetitively activates sequentially each stack, in order to let them execute/purge their tasks. Tasks stacks are launched into threads so that the user only has to append tasks and do not matter to check when they are terminated or not. Since all stacks are regrouped into one object, it is easier to link them, as shown in figure 10: for this example, the next tasks lists sequentially activates two Walking Tasks and a keyframe. As expected, the generated behaviour drives the agent from location 1, then 2 , then 3 and once the agent is arrived, make it applaud. In parallel, the visual attention of the agent is focusing on a car.

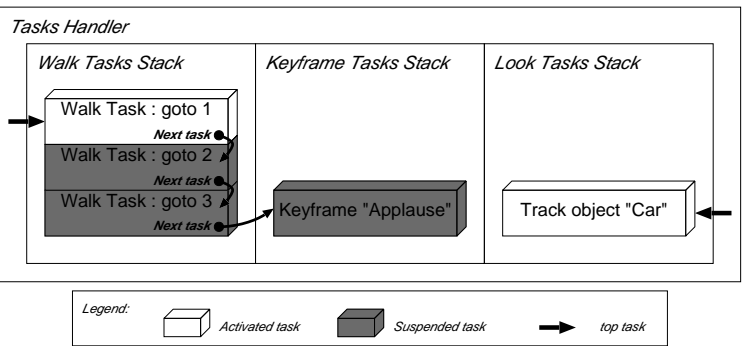

Figure 10: The Tasks Handler

\section{Results}

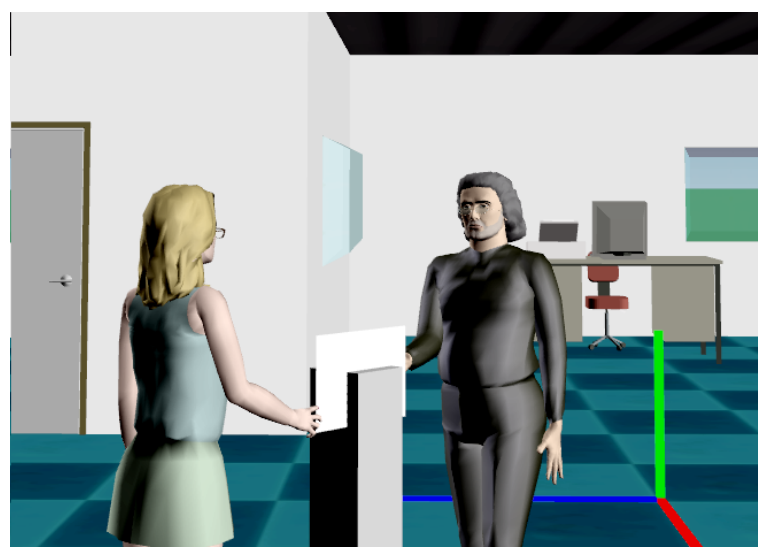

Figure 11: Two agents are exchanging an object.

Behavioural animation is not magic: without defining plans, agents are not able to act by themselves. Nevertheless, by sharing the same plans for conversing or delegating jobs depending on each others' roles, each agent only has to define how it performs its jobs, and this is only a matter of very few plans. For instance, agents in figure 11 are exchanging a sheet of paper, just after conversing and finding out that the Editor (on the right) can delegate the job (represented by the paper) to the Secretary. The Exchanger object is here shown as grey box. Plans in this case were common to both agents. The only difference between them was the role that they played during the simulation. 


\section{Conclusion and future work}

We have addressed in the paper the challenge of integrating 3D animation and behaviour simulation. The proposed system is separating in the low-level the physical simulation from the high-level decisions taking but it ensures that there is a communication established between both levels. Together with threads, this separation helps to manage concurrent processes without interlocking problems. We also presented how internal states and goals can be mixed within plans, and regarding the animation itself, how simultaneous gestures can combined using tasks stacks.

We are now developing a module for generating automatically context-dependent sentences, so that the system is able to produce the English text based on the semantic. This will be integrated in the system, together with an intelligent and automatic control of cameras. Finally, adding better emotions is also on the way.

\section{Acknowledgements}

This research was partly supported by the Federal Office for Education and Science in the framework of the SONG IST project.

\section{References}

1. P. Baerlocher and R. Boulic. Task priority formulations for the kinematic control of highly redundant articulated structures. In IEEE IROS' 98, pages 323-329, 1998.

2. R. Bindiganavale, W. Schuler, Allbeck J., Badler N., Joshi A., and M. Palmer. Dynamically altering agent behaviors using natural language instructions. In $\mathrm{Au}$ tonomous Agents 2000 Proceedings, 2000.

3. Grady Booch, Ivar Jacobson, James Rumbaugh, and Jim Rumbaugh. The Unified Modeling Language User Guide. Addison-Wesley, 1998.

4. C. Bordeux R. Boulic and D. Thalmann. An efficient and flexible perception pipeline for autonomous agents. In Proceedings of Eurographics' 99, pages 2330, 1999.

5. R. Boulic, P. Becheiraz, L. Emering, and D. Thalmann. Integration of motion control techniques for virtual human and avatar real-time animation. ACM Symposium on Virtual Reality Software and Technology, September 1997.

6. R. Brooks. A robust layered control system for a modbile robot. IEEE Journal of Robotics and Automation $R A-2,1986$.

7. A. Caicedo and D. Thalmann. Virtual humanoids: let them be autonomous without losing control. In The Fourth International Conference on Computer Graphics and Artificial Intelligence, 2000.
8. Justine Cassell, Catherine Pelachaud, Norman Badler, Mark Steedman, Brett Achorn, Tripp Bechet, Brett Douville, Scott Prevost, and Matthew Stone. Animated conversation: Rule-based generation of facial expression gesture and spoken intonation for multiple. In Andrew Glassner, editor, Proceedings of SIGGRAPH 94, pages 413-420. ACM Press, 1994.

9. John P. Granieri, Welton Becket, Barry D. Reich, Jonathan Crabtree, and Norman L. Badler. Behavioral control for real-time simulated human agents. 1995 Symposium on Interactive 3D Graphics, pages 173180, April 1995.

10. M. Kallmann and D. Thalmann. A behavioral interface to simulate agent-object interactions in real-time. In IEEE Computer Society Press, editor, Proceedings of Computer Animation 99, pages 138-146, 1999.

11. P. Maes. How to do the right thing. Connection Science Journal, 1:291-323, Dec 1989.

12. M. Mateas. An Oz-centric review of interactive drama and believable agents, 1997.

13. M. Minsky. The society of mind. Simon and Schuster, 1988.

14. Karen Moltenbrey. All the right moves. Computer Graphics Word, 22, October 1999.

15. Ken Perlin and Athomas Goldberg. Improv: A system for scripting interactive actors in virtual worlds. Proceedings of SIGGRAPH 96, pages 205-216, August 1996.

16. Python. http://www.python.org.

17. A. S. Rao and M. P. Georgeff. Modeling rational agents withing a bdi-architecture. In J. Allen, R. Fikes, and E. Sandewall, editors, Proceedings of the Third International Conference on Principles of Knowledge Representation and Reasoning. Morgan Kaufmann, 1991.

18. Dan Teven. Virtools' NeMo. Game Developer Magazine, September 1999.

19. N. Tinbergen. The study of Instinc. Oxford University Press, 1951. 